\title{
Illuminating changes in trace element geochemistry and mineralogy of bauxite residue during remediation
}

GraCe SCUllett-DEAN ${ }^{1}$, KATHERINE STOCKWELL ${ }^{1}$, Alex ChIU $^{1}$, TALitha SANTINI ${ }^{1}$

${ }^{1}$ School of Agriculture and Environment, The University of Western Australia, 35 Stirling Highway, 6009 WA, Australia

(*correspondence: grace.scullett-dean@research.uwa.edu.au)

Bauxite residue is the highly alkaline ( $\mathrm{pH} \sim 12)$ and saline tailings material produced during the Bayer process for alumina production. A relatively new approach to tailings management is in situ remediation, which aims to transform the residues into a soil-like medium, by reducing $\mathrm{pH}$, salinity and improving other unfavourable conditions, in order to establish vegetation directly into the residues. Our work focusses on guiding the transformation of bauxite residue into soil, using a variety of techniques spanning spatial scales, to model the advancement of soil formation. Fundamental investigations at a micro-scale will be used to guide practical in situ remediation technologies at a field (macro-) scale. Various analytical techniques are being used to model soil forming processes and will be key for identifying the optimal amendment combinations and the rates of remediation to improve tailings management.

Synchrotron X-ray diffraction (SXRD) was used to identify, quantify and model changes in residue mineralogy across depth and treatment type; certain minerals are expected to be mobilised during remediation from $\mathrm{pH} \sim 12$ to 6 . SXRD was also used to quantify trace metal substitution within residues. Mineral processing residues can contain trace metals such as As, Cr, Mo, V and $\mathrm{Zn}$, that are most likely associated with iron oxides, and can pose challenges to successful remediation.Isomorphically substituted trace metals may also be released under changing geochemical conditions. Synchrotron XRD was used to identify small shifts in peak position representing loss or incorporation of trace metals from mineral structures. The Rietveld method within TOPAS Academic was used to quantify sample mineralogy and trace metal incorporation. A mixed acid digest followed by Inductively Coupled Plasma Optical Emission Spectroscopy (ICP-OES) was used to quantify element concentrations in the residues. Results from this work will be used to build a more accurate remediation model and will contribute to improved tailings management within the industry. 